\title{
Introduction to the special issue from the European conference on service-oriented and cloud computing
}

\author{
Flavio De Paoli • Ernesto Pimentel
}

Published online: 4 November 2014

(c) Springer-Verlag London 2014

The 1st European Conference on Service-Oriented and Cloud Computing (ESOCC) was held in Bertinoro (Italy) on September 19-21, 2012. This was a first edition of a new conference series born on the past experience of the Steering Committee with the organization of the European Conference on Web Services (ECOWS) conference series. The beginning of this new series was decided to renew and broaden the original scope to provide a forum where researchers and practitioners can discuss the latest advances on the topics of service-oriented and cloud computing, and foster future collaborations in Europe and beyond.

The conference received 57 submissions of research papers and, after a rigorous peer review process that provided at least three reviews per paper, only 12 papers were accepted, yielding an acceptance rate of $21 \%$. In addition, three short papers and three posters were accepted. This allowed us to set up a high-quality program with papers giving important insights on web services and cloud computing, which have been clustered in four sessions on composition, security, modeling and adaptation. Among accepted papers, chairs and steering committee members selected five papers to be extended for this special issue. After an additional review process, four papers were finally accepted to represent the best of what the conference had to offer covering key topics in the field.

\footnotetext{
F. De Paoli

Department of Informatics, Systems and Communication, University of Milano-Bicocca,

Viale Sarca 336/14, 20126 Milan, Italy

e-mail:depaoli@disco.unimib.it

E. Pimentel $(\varangle)$

Department of Computer Science, Universidad de Málaga,

Boulevard Louis Pasteur 35, 29071 Málaga, Spain

e-mail: epimentel@uma.es
}

The paper "Efficient adaptive QoS-based service selection," by Lina Barakat, Simon Miles and Michael Luck, addresses the issue of service unavailability or contract violations, whereas services are running by proposing a reactive service selection algorithm, which adapts to dynamic changes in the environment while performing the selection, ensuring that the selected composite service is executable, satisfactory and optimal prior to execution. The effectiveness of the algorithm is demonstrated analytically, and empirically through a case study evaluation.

The paper "Orchestrating web services using Reo: from circuits and behaviors to automatically generated code," by Sung-Shik T. Q. Jongmans, Francesco Santini, Mahdi Sargolzaei, Farhad Arbab and Hamideh Afsarmanesh, discusses the issue of translating constructs specified with a purely declarative formal modeling language such as Reo into an executable piece of code whose operational semantics implements the modeled behavior, and which uses the usual WS technology stack (WSDL/SOAP) for interfacing with the outside world.

The paper "On expanding protocol conformance checking to exception handling," by Christian Heike, Wolf Zimmermann and Andreas Both, tackles the issue of conformance checking in presence of exceptions raised by composite services. Each service in the composition is associated with a behavioral contract expressed by a finite state machine that represents the order of operation calls. The proposed approach takes into account that the execution of service may raise an exception and allows the clients to react on the exception.

The paper "Formal modeling and analysis of resource management for cloud architectures: an industrial case study using Real-Time ABS," by Elvira Albert, Frank De Boer, Reiner Hähnle, Einar Broch Johnsen, Rudolf Schlatte, S. Lizeth Tapia Tarifa and Peter Y. H. Wong, reports on a 
case study evaluating the use of a modeling and simulation approach based on ABS, an object-based modeling language, extended by deployment information and non-functional aspects. The formal model addresses costs and quality of service, namely performance and resource consumption. Dynamic deployment strategies are discussed to ensure scalability under cost constraints on resource allocation.

This special section is the result of tremendous efforts from many volunteers in the community. We would like to thank the Steering and Program Committees for their work in reviewing the submissions to the conference in addition to helping identify a set of best papers from which the papers in this special session are drawn. Finally, this special section would not have been possible without the efforts of the anonymous reviewers who shared their time and expertize in carefully reviewing this selection of papers. 